PROCEEDINGS OF THE AMERICAN MATHEMATICAL SOCIETY

Volume 124, Number 3, March 1996

\title{
TOPOLOGICALLY CONJUGATE KLEINIAN GROUPS
}

\author{
KEN'ICHI OHSHIKA
}

(Communicated by Ron Stern)

\begin{abstract}
Two Kleinian groups $\Gamma_{1}$ and $\Gamma_{2}$ are said to be topologically conjugate when there is a homeomorphism $f: S^{2} \rightarrow S^{2}$ such that $f \Gamma_{1} f^{-1}=\Gamma_{2}$. It is conjectured that if two Kleinian groups $\Gamma_{1}$ and $\Gamma_{2}$ are topologically conjugate, one is a quasi-conformal deformation of the other. In this paper generalizing Minsky's result, we shall prove that this conjecture is true when $\Gamma_{1}$ is finitely generated and freely indecomposable, and the injectivity radii of all points of $\mathbf{H}^{3} / \Gamma_{1}$ and $\mathbf{H}^{3} / \Gamma_{2}$ are bounded below by a positive constant.
\end{abstract}

\section{INTRODUCTION}

Kleinian groups $\Gamma_{1}$ and $\Gamma_{2}$ are said to be topologically conjugate when there is a homeomorphism $f: S^{2} \rightarrow S^{2}$ such that $\Gamma_{2}=f \Gamma_{1} f^{-1}$. In particular, if $f$ can be taken to be a quasi-conformal homeomorphism, we say that $\Gamma_{1}$ and $\Gamma_{2}$ are quasi-conformally conjugate. It is conjectured that topologically conjugate Kleinian groups are also quasi-conformally conjugate. (See Minsky [4].) As quasi-conformal deformations of Kleinian groups are fairly well understood, if this conjecture is solved, it will help much to understand the topological property of the actions of Kleinian groups on $S^{2}$.

Marden proved that this conjecture is true (as a corollary of his isomorphism theorem) when the Kleinian group $\Gamma_{1}$ is geometrically finite ([3]). Minsky proved in [4], using his strong result on the rigidity of freely indecomposable Kleinian group with bounded injectivity radius, that when both groups are surface Kleinian groups such that there is a positive lower bound of the injectivity radii at all points of their quotient hyperbolic manifolds, then the conjecture is true. In this paper, we shall generalize Minsky's result to general freely indecomposable Kleinian groups with the same condition on the injectivity radius as above. Our result heavily depends on Minsky's theorem on the rigidity relative to the end invariant of freely indecomposable Kleinian group with bounded injectivity radius and is also motivated by his paper [4].

This paper was written during the author's stay at the University of Warwick for the symposium "Analytic and geometric aspects of hyperbolic spaces". The author would like to express his gratitude to the organizers of the symposium, Professors David Epstein and Caroline Series, for inviting him there and giving him much mathematical stimuli.

Received by the editors November 17, 1993.

1991 Mathematics Subject Classification. Primary 30F40, 57M50.

Key words and phrases. Kleinian group, topological conjugacy.

(C)1996 American Mathematical Society 


\section{Preliminaries}

Kleinian groups are discrete subgroups of the Lie group $P S L_{2}$ C. In this paper, we always assume that Kleinian groups are finitely generated and torsion free. As $P S L_{2} \mathbf{C}$ is the group of conformal automorphisms of $S^{2}$, a Kleinian group acts on $S^{2}$ conformally. On the other hand, as $P S L_{2} \mathbf{C}$ is also the full group of orientation preserving isometries of the hyperbolic 3-space $\mathbf{H}^{3}$ (whose ideal boundary is identified with $S^{2}$ and denoted by $S_{\infty}^{2}$ ), a Kleinian group $\Gamma$ acts on $\mathbf{H}^{3}$ discontinuously, and the quotient $\mathbf{H}^{3} / \Gamma$ is a complete hyperbolic 3-manifold.

We say two Kleinian groups $\Gamma_{1}$ and $\Gamma_{2}$ are topologically conjugate when there is a homeomorphism $f: S^{2} \rightarrow S^{2}$ such that $\Gamma_{2}=f \Gamma_{1} f^{-1}$. A homeomorphism $f: S^{2} \rightarrow S^{2}$ is said to be quasi-conformal when there is a function $\mu: S^{2} \rightarrow \mathbf{C}$ whose $L^{\infty}$-norm is less than 1 such that $f_{\bar{z}}=\mu f_{z}$ for any $z \in S^{2}$.

For a Kleinian group $\Gamma$ its limit set $\Lambda_{\Gamma} \subset S_{\infty}^{2}$ is the closure of the set of fixed points in $S_{\infty}^{2}$ of elements of $\Gamma$. The complement of $\Lambda_{\Gamma}$ is called the region of discontinuity of $\Gamma$. Let $C_{\Gamma}$ be the intersection of $\mathbf{H}^{3}$ and the convex hull of $\Lambda_{\Gamma}$ in $\mathbf{H}^{3} \cup S_{\infty}^{2}$. Then $C_{\Gamma}$ is $\Gamma$-invariant since $\Lambda_{\Gamma}$ is $\Gamma$-invariant. The quotient $C_{\Gamma} / \Gamma$ is a deformation retract of $\mathbf{H}^{3} / \Gamma$. A Kleinian group $\Gamma$ is said to be geometrically finite when $C_{\Gamma} / \Gamma$ has finite volume.

We say a Kleinian group $\Gamma$ is freely indecomposable when $\Gamma$ is not decomposed into a non-trivial free product of its subgroups. By Bonahon's theorem, freely indecomposable Kleinian groups are geometrically tame. For the definition of geometrical tameness, refer to Thurston [7] or Bonahon [1]. In particular, every end of $\mathbf{H}^{3} / \Gamma$ with a geometrically tame $\Gamma$ has a neighbourhood which is homeomorphic to the product of a closed surface and $\mathbf{R}$.

For an open 3-manifold $M$, we call a compact 3-submanifold $C \subset M$ a core when $C$ is a deformation retract of $M$. P. Scott proved that any 3-manifold with finitely generated fundamental group contains a core. If $\Gamma$ is freely indecomposable, a core of $\mathbf{H}^{3} / \Gamma$ is boundary-irreducible, i.e., every boundary component of $C$ is incompressible. In this case moreover, a core is unique up to isotopy.

Let $M$ be a hyperbolic 3-manifold. The injectivity radius at $z \in M$ is the infinimum of the length of a non-contractible loop based at $z$. We shall deal with hyperbolic 3-manifolds such that the injectivity radii at all points are bounded below by a positive constant. In particular, for such a hyperbolic 3-manifold $\mathbf{H}^{3} / \Gamma$, the Kleinian group $\Gamma$ has no parabolic elements, i.e., elements conjugate to matrices of the form $\left[\begin{array}{ll}1 & \lambda \\ 0 & 1\end{array}\right]$.

\section{The MAIN THEOREM AND ITS PROOF}

Theorem 1. Let $\Gamma_{1}$ and $\Gamma_{2}$ be freely indecomposable Kleinian groups. Assume that there is a lower bound $\epsilon>0$ of the injectivity radii at all points in $\mathbf{H}^{3} / \Gamma_{1}$ and $\mathbf{H}^{3} / \Gamma_{2}$. Suppose that there exists a homeomorphism $f: S^{2} \rightarrow S^{2}$ such that $f \Gamma_{1} f^{-1}=\Gamma_{2}$. Then there exists a quasi-conformal homeomorphism $\omega: S^{2} \rightarrow S^{2}$ such that $\omega \gamma \omega^{-1}=f \gamma f^{-1}$ for every $\gamma \in \Gamma_{1}$, hence especially $\omega \Gamma_{1} \omega^{-1}=\Gamma_{2}$.

We shall deduce Theorem 1 from the following theorem of Minsky [4]. We state his theorem in a form slightly different from the original for our convenience.

Theorem 2. (Minsky) Let $G_{1}$ and $G_{2}$ be freely indecomposable Kleinian groups. Assume that there is a lower bound $\epsilon>0$ of the injectivity radii at all points of $\mathbf{H}^{3} / G_{1}$ and $\mathbf{H}^{3} / G_{2}$. Suppose that there is a homeomorphism $h: \mathbf{H}^{3} / G_{1} \rightarrow$ 
$\mathbf{H}^{3} / G_{2}$ such that both $h$ and $h^{-1}$ map ending laminations to ending laminations. (In other words, if $\lambda$ is a measured lamination on a boundary component of a core $S$ which cannot be realized by a pleated surface homotopic to the inclusion $\iota: S \rightarrow \mathbf{H}^{3} / G_{1}$, then it is not realized by a pleated surface homotopic to $f \circ \iota$ either, and the same holds for $\iota^{\prime}: S \rightarrow \mathbf{H}^{3} / G_{2}$ and $f^{-1} \circ \iota^{\prime}$.) Then there is a quasi-conformal homeomorphism $w: S^{2} \rightarrow S^{2}$ such that $w g w^{-1}=h_{\#}(g)$ for every $g \in G_{1}$, hence especially $w G_{1} w^{-1}=G_{2}$.

We want to show that $\Gamma_{1}$ and $\Gamma_{2}$ in Theorem 1 satisfy the conditions in Theorem 2 above. For that, we shall first prove the following lemma which is an essential part of the proof.

Lemma 3. For $\Gamma_{1}$ and $\Gamma_{2}$ in Theorem 1 , there exists a homeomorphism $h: \mathbf{H}^{3} / \Gamma_{1}$ $\rightarrow \mathbf{H}^{3} / \Gamma_{2}$ such that $h_{\#}: \pi_{1}\left(\mathbf{H}^{3} / \Gamma_{1}\right) \cong \Gamma_{1} \rightarrow \pi_{1}\left(\mathbf{H}^{3} / \Gamma_{2}\right) \cong \Gamma_{2}$ induces an isomorphism equivalent to the conjugation by $f$.

Proof. By assumption, there is a homotopy equivalence $g: \mathbf{H}^{3} / \Gamma_{1} \rightarrow \mathbf{H}^{3} / \Gamma_{2}$ induced by the isomorphism $\Gamma_{2} \cong f \Gamma_{1} f^{-1}$. We have to prove that $g$ can be homotoped to a homeomorphism.

Since we assumed that both $\Gamma_{1}$ and $\Gamma_{2}$ are freely indecomposable, there are cores $C_{1}$ of $\mathbf{H}^{3} / \Gamma_{1}$ and $C_{2}$ of $\mathbf{H}^{3} / \Gamma_{2}$ such that every component of the complement of $C_{i}$ is homeomorphic to the product of the boundary component of $C_{i}$ facing the component and $\mathbf{R}$ for $i=1,2$. We shall prove that $g$ can be homotoped so that $g \mid C_{1}$ is a homeomorphism to $C_{2}$.

We can assume that $\bar{g}=g \mid C_{1}$ is a homotopy equivalence to $C_{2}$ because both $C_{1}$ and $C_{2}$ are cores. Therefore the point is to prove that $\bar{g}$ preserves the peripheral structure. Then by applying Waldhausen's theorem [8], we can prove that $\bar{g}$ is homotopic to a homeomorphism to $C_{2}$.

Now let $S$ be a boundary component of $C_{1}$. Then $S$ is incompressible since $\Gamma_{1}$ is freely indecomposable. We want to prove that $\bar{g} \mid S$ is homotopic to a map to a boundary component of $C_{2}$. Let $\Gamma^{S}$ be a subgroup of $\Gamma_{1}$ corresponding to $\pi_{1}(S)$. We shall consider the following two possibilities;

(1) $\Gamma^{S}$ is geometrically finite,

(2) $\Gamma^{S}$ is geometrically infinite.

(1) The case when $\Gamma^{S}$ is geometrically finite. Since $\Gamma^{S}$ corresponds to a peripheral subgroup of $\pi_{1}\left(C_{1}\right)$, there is a component $\Omega^{S}$ of the region of discontinuity of $\Gamma_{1}$ whose stabilizer is exactly $\Gamma^{S}$. Then $f \Omega^{S}$ is a component of the region of discontinuity of $\Gamma_{2}$ whose stabilizer is $f \Gamma^{S} f^{-1}$. It follows that $f \Gamma^{S} f^{-1}$ is a component subgroup, hence corresponds to a peripheral subgroup. Therefore $g_{\#} \pi_{1}(S)$ is a peripheral subgroup, which implies that $\bar{g} \mid S$ can be homotoped to a homeomorphism to a boundary component of $C_{2}$.

(2) The case when $\Gamma^{S}$ is geometrically infinite. Let $\Gamma_{2}^{S}$ be $f \Gamma^{S} f^{-1} \subset \Gamma_{2}$. We shall show that $\Gamma_{2}^{S}$ is also geometrically infinite. Suppose that $\Gamma_{2}^{S}$ is geometrically finite on the contrary. Then $\Gamma_{2}^{S}$ is a quasi-Fuchsian group, hence the limit set of $\Gamma_{2}^{S}$ is a Jordan curve $K$. Then as $\Gamma^{S}=f^{-1} \Gamma_{2}^{S} f$, the limit set of $\Gamma^{S}$ is also a Jordan curve $f^{-1}(K)$. This implies that $\Gamma^{S}$ is also quasi-Fuchsian, and contradicts our assumption. Thus $\Gamma_{2}^{S}$ is also geometrically infinite.

Since $\Gamma_{2}^{S}$ is freely indecomposable (because it is isomorphic to a surface group), it must be geometrically tame by Bonahon's theorem. Let $e$ be a geometrically infinite tame end of $\mathbf{H}^{3} / \Gamma_{2}^{S}$. Let $q: \mathbf{H}^{3} / \Gamma_{2}^{S} \rightarrow \mathbf{H}^{3} / \Gamma_{2}$ be the covering projection associated 
to the inclusion $\Gamma_{2}^{S} \subset \Gamma_{2}$. By Theorem 9.2.2 in Thurston [7] or Lemma 2.2 in Ohshika [5], there exists a neighbourhood $E$ of $e$ which is homeomorphic to $S \times \mathbf{R}$ such that $q \mid E$ is a finite-sheeted covering to its image. This implies that there exist an end $\bar{e}$ of $\mathbf{H}^{3} / \Gamma_{2}$ and its neighbourhood $\bar{E}$ homeomorphic to $\Sigma \times \mathbf{R}$ such that $q \mid S \times\{t\}$ covers $\Sigma \times\{t\}$. We can assume then that the component of $\mathbf{H}^{3} / \Gamma_{2}-C_{2}$ containing $\bar{e}$ is $\bar{E}$. Now, $g \mid S$ lifts to a homotopy equivalence $\tilde{g}: S \rightarrow \mathbf{H}^{3} / \Gamma_{2}^{S}$. We can homotope $g$ so that $\tilde{g}$ is a homeomorphism to $S \times\{t\} \subset E$. It follows that $g \mid S=q \circ \tilde{g}$ is homotopic to a finite-sheeted covering map onto $\Sigma \times\{t\}$, hence to a covering map onto a boundary component of $C_{2}$.

Thus in either case $\bar{g} \mid S$ is homotopic to a covering map to a boundary component of $C_{2}$. Although the homotopy is in $\mathbf{H}^{3} / \Gamma_{2}$, we can assume that it is in $C_{2}$ as $C_{2}$ is a core. Hence $\bar{g}: C_{1} \rightarrow C_{2}$ can be homotoped so that $g \mid \partial C_{1}$ is a covering map to the union of components of $\partial C_{2}$. Since $\bar{g}$ is a homotopy equivalence, this implies that $\bar{g}$ is homotopic to a homeomorphism to $C_{2}$ preserving $\bar{g} \mid \partial C_{1}$ by Waldhausen's theorem [8] (see also Hempel [2], Corollary 13.7). Obviously this extends to a homotopy from $g$ to a homeomorphism from $\mathbf{H}^{3} / \Gamma_{1}$ to $\mathbf{H}^{3} / \Gamma_{2}$ as each component of $\mathbf{H}^{3} / \Gamma_{i}-C_{i}$ is the product of a surface and $\mathbf{R}$.

Proof of Theorem 1. By Lemma 3, there is a homeomorphism $h: \mathbf{H}^{3} / \Gamma_{1} \rightarrow \mathbf{H}^{3} / \Gamma_{2}$ which induces an isomorphism equivalent to the conjugation by $f$. It remains to prove that $h$ and $h^{-1}$ map ending laminations to ending laminations.

Let $e$ be an end of $\mathbf{H}^{3} / \Gamma_{1}$ for which $\lambda$ is an ending lamination. Let $S_{1}$ be a boundary component of the core $C_{1}$ of $\mathbf{H}^{3} / \Gamma_{1}$ facing the end $e$. We can assume that $\lambda$ lies on $S_{1}$. The homeomorphism $h$ maps $C_{1}$ to a core $h\left(C_{1}\right)=C_{2}$ of $\mathbf{H}^{3} / \Gamma_{2}$. Let $S_{2}$ be $h\left(S_{1}\right)$. Let $\Gamma_{1}^{S}$ be a subgroup of $\Gamma_{1}$ corresponding to $\pi_{1}\left(S_{1}\right)$ and let $\Gamma_{2}^{S}$ be $f \Gamma_{1}^{S} f^{-1}$. The homeomorphism $h$ is covered by a homeomorphism $\tilde{h}: \mathbf{H}^{3} / \Gamma_{1}^{S} \rightarrow \mathbf{H}^{3} / \Gamma_{2}^{S}$. The surfaces $S_{1}$ and $S_{2}$ are lifted homeomorphically to $\tilde{S}_{1} \subset \mathbf{H}^{3} / \Gamma_{1}^{S}$ and $\tilde{S}_{2} \subset \mathbf{H}^{3} / \Gamma_{2}^{S}$ such that $\tilde{h}\left(\widetilde{S}_{1}\right)=\widetilde{S}_{2}$.

Let $\tilde{\lambda}$ be a homeomorphic lift of $\lambda$ into $\tilde{S}_{1}$. Let $p_{1}: \mathbf{H}^{3} / \Gamma_{1}^{S} \rightarrow \mathbf{H}^{3} / \Gamma_{1}$ and $p_{2}: \mathbf{H}^{3} / \Gamma_{2}^{S} \rightarrow \mathbf{H}^{3} / \Gamma_{2}$ be the covering projections associated to the inclusions. As $f \Gamma_{1}^{S} f^{-1}=\Gamma_{2}^{S}$, by Theorem $\mathrm{E}$ in Minsky [4], the topological rigidity for surface groups, $\tilde{h}(\tilde{\lambda})$ is an ending lamination, which means that there is no pleated surface homotopic to the inclusion of $\tilde{S}_{2} \subset \mathbf{H}^{3} / \Gamma_{2}^{S}$ realizing $\tilde{h}(\tilde{\lambda})$. If $h(\lambda)$ were not an ending lamination, there would be a pleated surface in $\mathbf{H}^{3} / \Gamma_{2}$ homotopic to the inclusion of $S_{2}$ realizing $h(\lambda)$, which would be lifted to a pleated surface in $\mathbf{H}^{3} / \Gamma_{2}^{S}$ homotopic to the inclusion of $\tilde{S}_{2}$ realizing $\tilde{h}(\tilde{\lambda})$. This is a contradiction. Therefore $h(\lambda)$ is also an ending lamination.

By the same argument we can prove that if $\mu \subset S_{2}$ is an ending lamination, so is $h^{-1}(\mu) \subset S_{1}$. Thus all the conditions of Theorem 2 are satisfied for our $\Gamma_{1}$ and $\Gamma_{2}$ and it follows from Theorem 2 that there exists a quasi-conformal homeomorphism $\omega: S^{2} \rightarrow S^{2}$ such that $\omega \gamma \omega^{-1}=h_{\#}(\gamma)=f \gamma f^{-1}$. This completes the proof of Theorem 1 .

\section{REFERENCES}

1. F. Bonahon, Bouts des variétés hyperboliques de dimension 3, Ann. of Math. 124 (1986), 71-158. MR 88c:57013

2. J. Hempel, 3-manifolds, Ann. Math. Studies 86, Princeton Univ. Press, Princeton New Jersey, (1976). MR 54:3702 
3. A. Marden, The geometry of finitely generated Kleinian groups, Ann. of Math., 99 (1974), 383-462. MR 50:2485

4. Y. Minsky, On rigidity, limit sets, and end invariants of hyperbolic 3-manifolds, J. Amer. Math. Soc. 7 (1994), 539-588. MR 94m:57029

5. K. Ohshika, Geometric behaviour of Kleinian groups on boundaries for deformation spaces, Quart. J. Math. Oxford (2), 43 (1992), 97-111. MR 93a:57018

6. G. P. Scott, Compact submanifolds of 3-manifolds, J. London Math. Soc. 7 (1973), 246-250. MR 48:5080

7. W. Thurston, The geometry and topology of 3-manifolds, lecture notes, Princeton Univ.

8. F. Waldhausen, On irreducible 3-manifolds which are sufficiently large, Ann. of Math. 87 (1968), 56-88. MR 36:7146

Department of Mathematics, Tokyo Institute of Technology, Oh-OKayama, MeguroKU, TOKYO 152, JAPAN

E-mail address: ohshika@math.titech.ac.jp 